\title{
Longitudinal fracture of inhomogeneous beams with material non-linearity: An analytical investigation
}

\author{
V. Rizov ${ }^{a, *}$ \\ ${ }^{a_{\text {Department }}}$ of Technical Mechanics, University of Architecture, Civil Engineering and Geodesy, 1 Chr. Smirnensky blvd., 1046 - \\ Sofia, Bulgaria
}

Received 13 September 2018; accepted 24 April 2020

\begin{abstract}
This paper describes an analytical approach for analysing of longitudinal fracture in beam structures which exhibit smooth material inhomogeneity along the height and length. A solution to the strain energy release rate is derived for a general loading scheme assuming non-linear mechanical behaviour of the material. The Ramberg-Osgood stress-strain relation is used to model the material non-linearity. It is assumed that the three material properties which are involved in the Ramberg-Osgood stress-strain relation vary continuously along the height and length of the beam (exponential laws are applied for describing the distribution of these properties along the height and length of the beam). The solution derived is valid for a longitudinal crack located arbitrary along the beam height. It is shown that the approach developed can be applied for evaluating the effects of material inhomogeneity along the height and length of the beam, non-linear mechanical behaviour of the material, crack location along the beam height and the crack length on the longitudinal fracture.
\end{abstract}

(c) 2020 University of West Bohemia. All rights reserved.

Keywords: material inhomogeneity, beam structure, longitudinal fracture, material non-linearity

\section{Introduction}

The properties of the inhomogeneous materials are functions of coordinates. This fact complicates significantly the fracture analysis of structural members and components made of inhomogeneous materials. Typical examples for inhomogeneous materials are the functionally graded materials which are used as advanced structural materials in various engineering applications mainly because the spatial variation of their properties can be tailored to meet the requirements for different parts of a structural member $[1,4,7,8]$. Fracture of inhomogeneous materials is an important subject $[2,3,6,9,10,16,17]$. Since some inhomogeneous materials, such as functionally graded materials, can be built up layer by layer [1], there is a high probability of appearance of longitudinal cracks between layers. Thus, longitudinal fracture analysis of inhomogeneous materials and structures is an important task of the present-day fracture mechanics. Beside the material inhomogeneity, another characteristic feature which should be taken into account in longitudinal fracture analyses is the non-linear mechanical behaviour of inhomogeneous materials.

Recently, various analyses of longitudinal cracks in inhomogeneous (functionally graded) beam structures have been developed by the author assuming non-linear mechanical behaviour of the material [11-14]. The fracture behaviour has been studied in terms of the strain energy release rate. It has been assumed that only one material property (usually, the modulus of elasticity) varies continuously in the beam cross-section.

\footnotetext{
*Corresponding author. Tel.: +(359-2) 96352 45/664, e-mail: v_rizov_fhe@uacg.bg. https://doi.org/10.24132/acm.2020.484
} 
The basic aim of the present paper is to peform an analytical investigation of longitudinal fracture of inhomogeneous beams under a genearl loading scheme by applying the RambergOsgood constitutive law for modelling non-linear elastic mechanical behaviour of the material assuming that the three material properties which are involved in the constitutive law vary smoothly along the thickness and longitudinal directions of the beam. Exponential laws are used for describing the continuous variation of the material properties in height and length directions. An analytical solution to the strain energy release rate is derived by analyzing the energy balance. The beams are subjected to static loading. It should be noted that the present analysis is based on the small strains assumption. The Euler-Bernoulli beam theory is applied. The beams under consideration have rectangular cross-section. The solution derived is used to investigate the effect of various geometrical and material parameters on the longitudinal fracture behaviour.

\section{Analysis of general loading scheme}

An inhomogeneous beam with a longitudinal crack of length, $a$, is shown in Fig. 1.

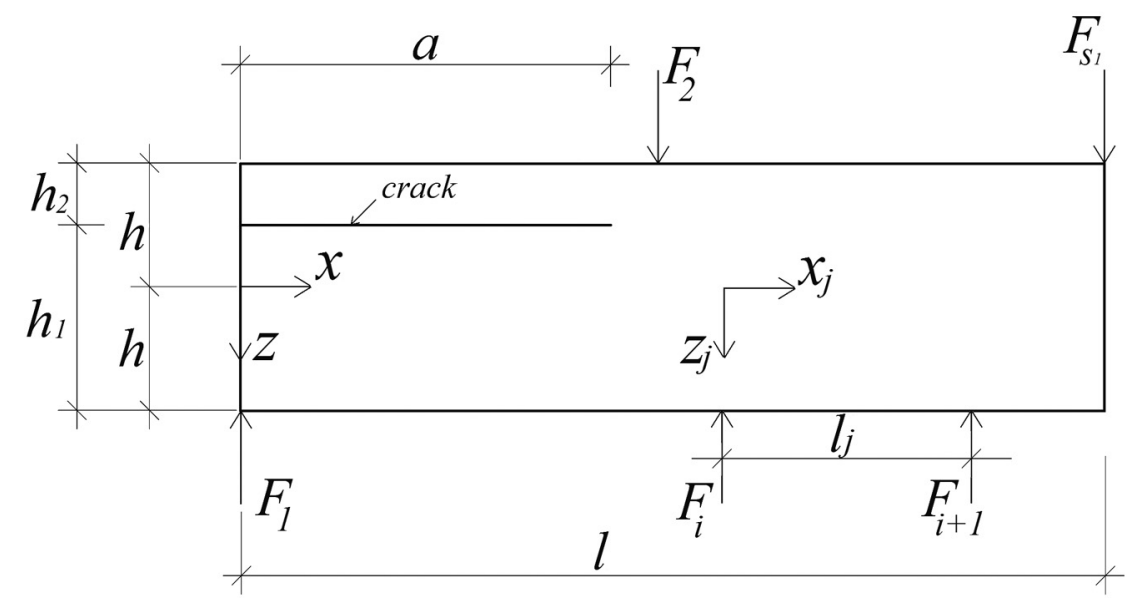

Fig. 1. Inhomogeneous beam containing a longitudinal crack under general loading

The two crack arms have different heights denoted by $h_{1}$ and $h_{2}$ for the lower and upper crack arms, respectively. The width and height of the beam cross-section are $b$ and $2 h$. The beam is in equilibrium under system of transversal forces $F_{i}$. General solution to the strain energy release rate $G$ is derived by considering the balance of the energy. For this purpose, the balance of the energy is written as [10]

$$
\sum_{i=1}^{s_{1}} F_{i} \delta w_{i}=\sum_{j=1}^{s_{2}} \frac{\partial U_{j}}{\partial a} \delta a+G b \delta a,
$$

where $s_{1}$ is the number of forces, $\delta a$ is a virtual change of the crack length, $\delta w_{i}$ is the increase of the vertical displacement of the application point of $F_{i}, s_{2}$ is the number of portions of the beam, $U_{j}$ is the strain energy cumulated in the $j$-th beam portion. From (1), the strain energy release rate is expressed as

$$
G=\sum_{i=1}^{s_{1}} \frac{F_{i}}{b} \frac{\partial w_{i}}{\partial a}-\frac{1}{b} \sum_{j=1}^{s_{2}} \frac{\partial U_{j}}{\partial a} .
$$


By applying the integrals of Maxwell-Mohr [15], the vertical displacement of the application pint of $F_{i}$ is written as

$$
w_{i}=\sum_{j=1}^{s_{2}} \int \kappa_{j} \overline{M_{j}} \mathrm{~d} x,
$$

where $\kappa_{j}$ is the curvature of the $j$-th beam portion, $\overline{M_{j}}$ is the bending moment in the $j$-th portion of the beam induced by the unit loading, $x$ is the longitudinal centroidal axis of the beam.

The strain energy cumulated in the beam is found as

$$
U=\sum_{j=1}^{s_{2}} U_{j}
$$

where strain energy $U_{j}$ in the $j$-th portion of the beam is obtained as

$$
U_{j}=b \int_{0}^{l_{j}} \int_{-h}^{h} u_{0 j} \mathrm{~d} x_{j} \mathrm{~d} z_{j} .
$$

In formula (5), $l_{j}$ is the length of the $j$-th portion of the beam (Fig. 1), $x_{j}$ and $z_{j}$ are the longitudinal and the vertical centroidal axes in the $j$-th portion of the beam, $u_{0 j}$ is the strain energy density in the same beam portion. The cross-section of the $j$-th portion of the beam is shown in Fig. 2.

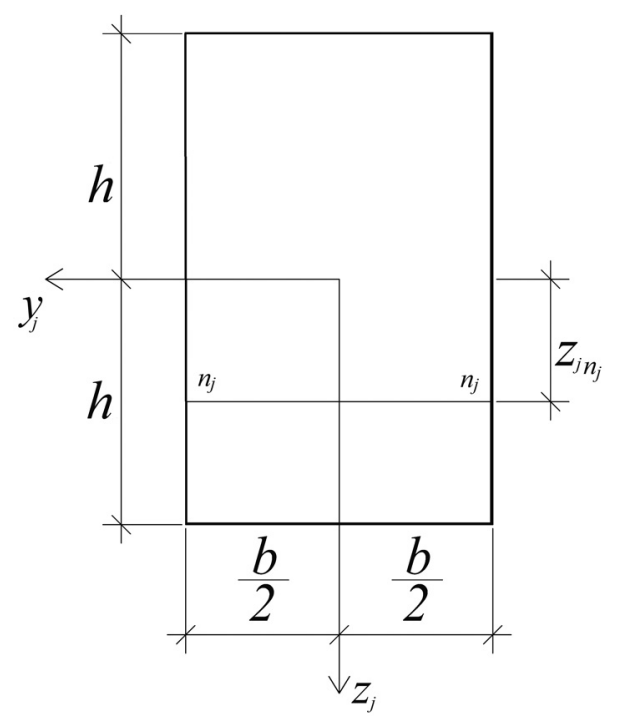

Fig. 2. Cross-section of the of $j$-th portion of the beam (the position of the neutral axis is denoted by $\left.n_{j}-n_{j}\right)$

By substituting of (3)-(5) in (2), one obtains the following expression for the strain energy release rate:

$$
G=\frac{1}{b} \sum_{i=1}^{s_{1}} F_{i} \frac{\partial}{\partial a}\left(\sum_{j=1}^{s_{2}} \int \kappa_{i} \overline{M_{j}} \mathrm{~d} x\right)-\sum_{j=1}^{s_{2}} \frac{\partial}{\partial a}\left(\int_{0}^{l_{j}} \int_{-h}^{h} u_{0 j} \mathrm{~d} x \mathrm{~d} z_{j}\right) .
$$

The non-linear elastic behaviour of the material is treated by using the Ramberg-Osgood stress-strain relation [5]

$$
\varepsilon_{j}=\frac{\sigma_{j}}{E_{j}}+\left(\frac{\sigma_{j}}{H_{j}}\right)^{\frac{1}{n_{j}}}
$$


where $\varepsilon_{j}$ is the distribution of the lengthwise strains, $\sigma_{j}$ is the distribution of the normal stresses, $E_{j}$ is the modulus of elasticity, $H_{j}$ and $n_{j}$ are material properties in the $j$-th portion of the beam.

The strain energy density that is involved in (6) is calculated by the following formula [9]:

$$
u_{0 j}=\frac{\sigma_{j}^{2}}{2 E_{j}}+\frac{\sigma_{j}^{\frac{1+n_{j}}{n_{j}}}}{\left(1+n_{j}\right) H_{j}^{\frac{1}{n_{j}}}} .
$$

In order to carry-out the integration in (6), the normal stress $\sigma_{j}$ that is involved in the expression for the strain energy density (8) has to be presented as a function of $z_{j}$. It is obvious that $\sigma_{j}$ can not be determined explicitly from the Ramberg-Osgood equation (7). Therefore, $\sigma_{j}$ is expanded in series of Maclaurin by keeping the first three members

$$
\sigma_{j}\left(z_{j}\right) \approx \sigma_{j}(0)+\frac{\sigma_{j}^{\prime}(0)}{1 !} z_{j}+\frac{\sigma_{j}^{\prime \prime}(0)}{2 !} z_{j}^{2} .
$$

Formula (9) is rewritten as

$$
\sigma_{j}\left(z_{j}\right) \approx \varphi_{0}+\varphi_{1} z_{j}+\varphi_{2} z_{j}^{2}
$$

where the coefficients $\varphi_{0}, \varphi_{1}$ and $\varphi_{2}$ are derived in the following manner. First, the distribution of $\varepsilon$ along the height of the lower crack arm is written as

$$
\varepsilon_{j}=\kappa_{j}\left(z_{j}-z_{j n_{j}}\right)
$$

where $z_{j n_{j}}$ is the coordinate of the neutral axis (Fig. 2). It should be noted that the neutral axis shifts from the centroid since the material exhibits inhomogeneity along the beam height. Formula (11) follows from the fact that validity of the Bernoulli's hypothesis for plane sections is assumed in the present paper.

Further, by substituting of (10) and (11) in (7), one obtains

$$
\kappa_{j}\left(z_{j}-z_{1 n_{j}}\right)=\frac{\varphi_{0}+\varphi_{1} z_{j}+\varphi_{2} z_{j}^{2}}{E_{j}}+\left(\frac{\varphi_{0}+\varphi_{1} z_{j}+\varphi_{2} z_{j}^{2}}{H_{j}}\right)^{\frac{1}{n_{j}}} .
$$

By substituting of $z_{j}=0$ in (12), one derives

$$
-z_{j n_{j}} \kappa_{j}=\frac{\varphi_{0}}{E_{j}}+\frac{\varphi_{0}^{\frac{1}{n_{j}}}}{H_{j}^{\frac{1}{n_{j}}}} .
$$

By differentiating of (12) with respect to $z_{j}$ and substituting of $z_{1}=0$, one obtains

$$
\kappa_{j}=\frac{\varphi_{1} E_{j}-\varphi_{0} E_{j}^{\prime}}{E_{j}^{2}}+\frac{\varphi_{0}^{m_{j}}}{H_{j}^{m_{j}}}\left[m_{j}^{\prime}\left(\ln \varphi_{0}-\ln H_{j}\right)+m_{j}\left(\frac{\varphi_{1}}{\varphi_{0}}-\frac{H_{j}^{\prime}}{H_{j}}\right)\right] .
$$

By substituting of $z_{j}=0$ in the second derivative of (12) with respect to $z_{1}$, one arrives at

$$
\begin{aligned}
0= & \frac{2 \varphi_{2} E_{j}-\varphi_{0} E_{j}^{\prime \prime}}{E_{j}^{2}}-\frac{\left(\varphi_{1} E_{j}-\varphi_{0} E_{j}^{\prime}\right) 2 E_{j}^{\prime}}{E^{3}}+ \\
& \frac{\varphi_{0}^{m_{j}}}{H_{j}^{m_{j}}}\left[m_{j}^{\prime}\left(\ln \varphi_{0}-\ln H_{j}\right)+m_{j}\left(\frac{\varphi_{1}}{\varphi_{0}}-\frac{H_{j}^{\prime}}{H_{j}}\right)\right]^{2}+ \\
& \frac{\varphi_{0}^{m_{j}}}{E_{j}^{m_{j}}}\left\{m_{j}^{\prime \prime}\left(\ln \varphi_{0}-\ln H_{j}\right)+2 m_{j}^{\prime}\left(\frac{\varphi_{1}}{\varphi_{0}}-\frac{H_{j}^{\prime}}{H_{j}}\right)+m_{j}\left[-\frac{\varphi_{1}^{2}}{\varphi_{0}^{2}}+\frac{2 \varphi_{2}}{\varphi_{0}}+\frac{\left(H_{j}^{\prime}\right)^{2}}{H_{j}^{2}}-\frac{H_{j}^{\prime \prime}}{H_{j}}\right]\right\},
\end{aligned}
$$


where

$$
\begin{aligned}
m & =\frac{1}{n} \\
m^{\prime} & =\left(\frac{1}{n}\right)^{\prime}, \\
m^{\prime \prime} & =\left(\frac{1}{n}\right)^{\prime \prime}
\end{aligned}
$$

It should be noted that $E^{\prime}, E^{\prime \prime}, H^{\prime}, H^{\prime \prime}, m^{\prime}$ and $m^{\prime \prime}$ are the first and second derivatives of $E, H$ and $m$ with respect to $z_{1}$ at $z_{1}=0$.

In equations (13)-(15) there are five unknowns $\varphi_{0}, \varphi_{1}, \varphi_{2}, \kappa_{j}$ and $z_{j n_{j}}$. Two other equations are constructed by considering the equilibrium of the elementary forces in the cross-section of the $j$-th portion of the beam

$$
\begin{aligned}
& N_{j}=b \int_{-h}^{h} \sigma_{j} \mathrm{~d} z_{j}, \\
& M_{j}=b \int_{-h}^{h} \sigma_{j} z_{j} \mathrm{~d} z_{j},
\end{aligned}
$$

where $N_{j}$ and $M_{j}$ are the axial force and the bending moment, respectively.

By substituting of (10) in (19) and (20), one derives

$$
\begin{aligned}
& N_{j}=b\left(2 \varphi_{0} h+\varphi_{2} \frac{2 h^{3}}{3}\right), \\
& M_{j}=b \varphi_{1} \frac{2 h^{3}}{3} .
\end{aligned}
$$

Equations (13)-(15) and (21)-(22) can be solved with respect to $\varphi_{0}, \varphi_{1}, \varphi_{2}, \kappa_{j}$ and $z_{j n_{j}}$ for each portion of the beam.

\section{Example}

Fig. 3 shows an inhomogeneous beam having a longitudinal crack of length $a$. The crack is located arbitrary along the beam height. The heights of the lower and upper crack arms are denoted by $h_{1}$ and $h_{2}$, respectively. The beam is supported by a roller at point III and a $Q$-apparatus in the free end of the lower crack arm.

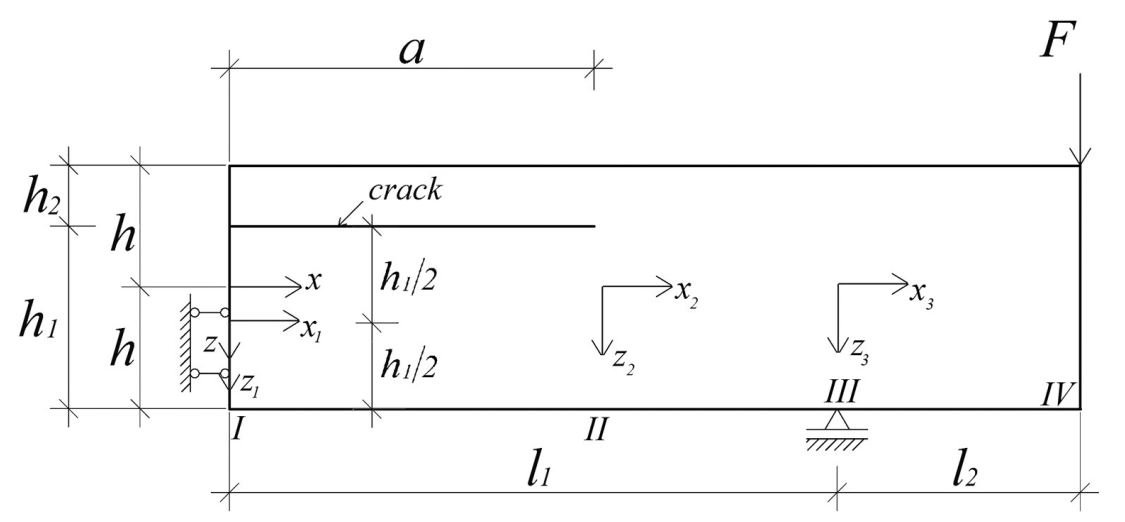

Fig. 3. Geometry and loading of inhomogeneous beam containing a longitudinal crack 
A vertical force $F$ is applied at the right-hand end of the beam. It should be mentioned that the crack is located in beam portion $I-I I$ that is loaded in pure bending (Fig. 3). The width and height of the beam are denoted by $b$ and $2 h$, respectively. The length of the beam is $l_{1}+l_{2}$. The lower crack arm has the following boundaries: $x_{1}=0, x_{1}=a, z_{1}=-h_{1} / 2$ and $z_{1}=h_{1} / 2$. The boundaries of beam portion II-III are $x_{2}=0, x_{2}=l_{1}-a, z_{2}=-h / 2$ and $z_{2}=h / 2$. The beam portion $I I I-I V$ has the following boundaries: $x_{3}=0, x_{3}=l_{2}, z_{3}=-h / 2$ and $z_{3}=h / 2$. The coordinate systems $x_{1} z_{1}, x_{2} z_{2}$ and $x_{3} z_{3}$ are shown in Fig. 3. It is assumed that the beam exhibits smooth material inhomogeneity along the thickness and longitudinal directions (the three material properties which are involved in the Ramberg-Osgood equation vary continuously along the thickness and longitudinal directions of the beam). The strain energy release rate is obtained by using the solution (6) for the general loading scheme developed in the prevoius section of the paper. By applying (3), the vertical displacement of the right-hand end of the beam is written as

$$
w=\int_{0}^{a} \kappa_{1} l_{2} \mathrm{~d} x+\int_{a}^{l_{1}} \kappa_{2} l_{2} \mathrm{~d} x+\int_{l_{1}}^{l_{1}+l_{2}} \kappa_{3}\left(l_{1}+l_{2}-x_{4}\right) \mathrm{d} x,
$$

where $\kappa_{1}, \kappa_{2}$ and $\kappa_{3}$ are the curvatures of the lower crack arm and beam portions II-III and $I I I-I V$, respectively. The $x$-axis is defined in Fig. 3.

The continuous variation of $E, H$ and $n$ along the thickness direction is expressed by the following exponential laws:

$$
\begin{aligned}
& E=E_{0} \mathrm{e}^{f_{1} \frac{z+h}{2 h}}, \\
& H=H_{0} \mathrm{e}^{f_{2} \frac{z+h}{2 h}}, \\
& n=n_{0} \mathrm{e}^{f_{3} \frac{z+h}{2 h}},
\end{aligned}
$$

where

$$
-h \leq z \leq h
$$

In (24)-(26) $E_{0}, H_{0}$ and $n_{0}$ are the values, respectively, of $E, H$ and $n$ at the upper surface of the beam, $f_{1}, f_{2}$ and $f_{3}$ are material properties which control the material gradient along the thickness. The $z$-axis is defined in Fig. 3.

The continuous variations of $E_{0}, H_{0}$ and $n_{0}$ along the beam length are written as

$$
\begin{aligned}
E_{0} & =E_{0 L} \mathrm{e}^{r_{1} \frac{x}{l_{1}+l_{2}}}, \\
H_{0} & =H_{0 L} \mathrm{e}^{r_{2} \frac{x}{l_{1}+l_{2}}}, \\
n_{0} & =n_{0 L} \mathrm{e}^{r_{3} \frac{x}{l_{1}+l_{2}}},
\end{aligned}
$$

where

$$
0 \leq x \leq l_{1}+l_{2}
$$

In (28)-(30) $E_{0 L}, H_{0 L}$ and $n_{0 L}$ are the values, respectively, of $E_{0}, H_{0}$ and $n_{0}$ at the left-hand end of the beam, $r_{1}, r_{2}$ and $r_{3}$ are material properties which control the material gradient along the longitudinal direction.

In order to describe the distributions of $E, H$ and $n$ along the thickness direction of the lower crack arm, formulae (24)-(26) are re-written as

$$
\begin{aligned}
E & =E_{0} \mathrm{e}^{g_{1} z_{1}+q_{1}}, \\
H & =H_{0} \mathrm{e}^{g_{2} z_{1}+q_{2}}, \\
n & =n_{0} \mathrm{e}^{g_{3} z_{1}+q_{3}},
\end{aligned}
$$


where

$$
\begin{aligned}
-\frac{h_{1}}{2} & \leq z_{1} \leq \frac{h_{1}}{2}, \\
g_{1} & =\frac{f_{1}}{2 h}, \\
q_{1} & =g_{1}\left(h-\frac{h_{1}}{2}\right)+\frac{f_{1}}{2}, \\
g_{2} & =\frac{f_{2}}{2 h}, \\
q_{2} & =g_{2}\left(h-\frac{h_{1}}{2}\right)+\frac{f_{2}}{2}, \\
g_{3} & =\frac{f_{3}}{2 h}, \\
q_{3} & =g_{3}\left(h-\frac{h_{1}}{2}\right)+\frac{f_{3}}{2} .
\end{aligned}
$$

The coordinate system $x_{1} z_{1}$ in the lower crack arm is shown in Fig. 3. The values of $E, H$ and $n$ and their derivatives involved in (13)-(15) are found as

$$
\begin{aligned}
E & =E_{0} \mathrm{e}^{q_{1}}, \\
E^{\prime} & =E_{0} \mathrm{e}^{q_{1}} g_{1}, \\
E^{\prime \prime} & =E_{0} \mathrm{e}^{q_{1}} g_{1}^{2}, \\
H & =H_{0} \mathrm{e}^{q_{2}}, \\
H^{\prime} & =H_{0} \mathrm{e}^{q_{2}} g_{2}, \\
H^{\prime \prime} & =H_{0} \mathrm{e}^{q_{2}} g_{2}^{2}, \\
m & =\frac{1}{n_{0} \mathrm{e}^{q_{3}}}, \\
m^{\prime} & =-\frac{g_{3}}{n_{0} \mathrm{e}^{q_{3}}}, \\
m^{\prime \prime} & =\frac{g_{3}^{2}}{n_{0} \mathrm{e}^{q_{3}}} .
\end{aligned}
$$

The axial force $N_{1}$ and the bending moment $M_{1}$ in the lower crack arm are determined in the following way. First, by using the equations for equlibrium of the beam

$$
\begin{aligned}
\sum F_{z i} & =0, \\
\sum M_{I I I} & =0,
\end{aligned}
$$

the vertical reaction $V_{I I I}$ in the roller at point $I I I$ and the bending moment $M_{L}$ in the $Q$-aparatus at the free end of the lower crack arm are found as

$$
\begin{aligned}
V_{I I I} & =F, \\
M_{L} & =F l_{2} .
\end{aligned}
$$

Therefore, the axial force and the bending moment in the lower crack arm are written as

$$
\begin{aligned}
& N_{1}=0, \\
& M_{1}=F l_{2} .
\end{aligned}
$$


Equations (13)-(15) and (21)-(22) are solved with respect to $\varphi_{0}, \varphi_{1}, \varphi_{2}, \kappa_{1}$ and $z_{1 n_{1}}$. It should be mentioned that these equations can be applied to determine $\varphi_{0}, \varphi_{1}, \varphi_{2}, \kappa_{1}$ and $z_{1 n_{1}}$ in any cross-section of the lower crack arm. For this purpose, $E_{0}, H_{0}$ and $n_{0}$ have to be calculated by (28)-(30) for the abscissa $x$ of the cross-section under consideration. The normal stress in the lower crack arm is obtained by (10). The normal stresses in the beam portions $I I-I I I$ and $I I I-I V$ are found analogically.

The strain energies in the lower crack arm and beam portions $I I-I I I$ and $I I I-I V$ are written as

$$
\begin{aligned}
& U_{1}=b \int_{0}^{a} \int_{-\frac{h_{1}}{2}}^{\frac{h_{1}}{2}} u_{01} \mathrm{~d} x \mathrm{~d} z_{1}, \\
& U_{2}=b \int_{a}^{l_{1}} \int_{-h}^{h} u_{02} \mathrm{~d} x \mathrm{~d} z_{2}, \\
& U_{3}=b \int_{l_{1}}^{l_{1}+l_{2}} \int_{-h}^{h} u_{03} \mathrm{~d} x \mathrm{~d} z_{3},
\end{aligned}
$$

respectively.

By substituting of (23), (57), (58) and (59) in (6), one obtains the following solution to the strain energy releaese rate for the longitudinal crack in the beam configuration shown in Fig. 3:

$$
G=\frac{F}{b} l_{2}\left(\kappa_{1}-\kappa_{2}\right)-\left(\int_{-\frac{h_{1}}{2}}^{\frac{h_{1}}{2}} u_{01} \mathrm{~d} z_{1}-\int_{-h}^{h} u_{02} \mathrm{~d} z_{2}\right),
$$

where $\kappa_{1}, \kappa_{2}, u_{01}$ and $u_{02}$ are obtained by (13)-(15) and (21)-(22) at $x=a$.

It should be noted that the strain energy releae rate is analyzed also by keeping more than three members in the series of Maclaurin (9). The results obtained are very close to these derived by keeping three members (the difference is less than $2 \%$ ).

\section{Parametric study}

The solution to the strain energy release rate (60) is applied to perform a parametric study of longitudinal fracture in the inhomogeneous beam shown in Fig. 3.

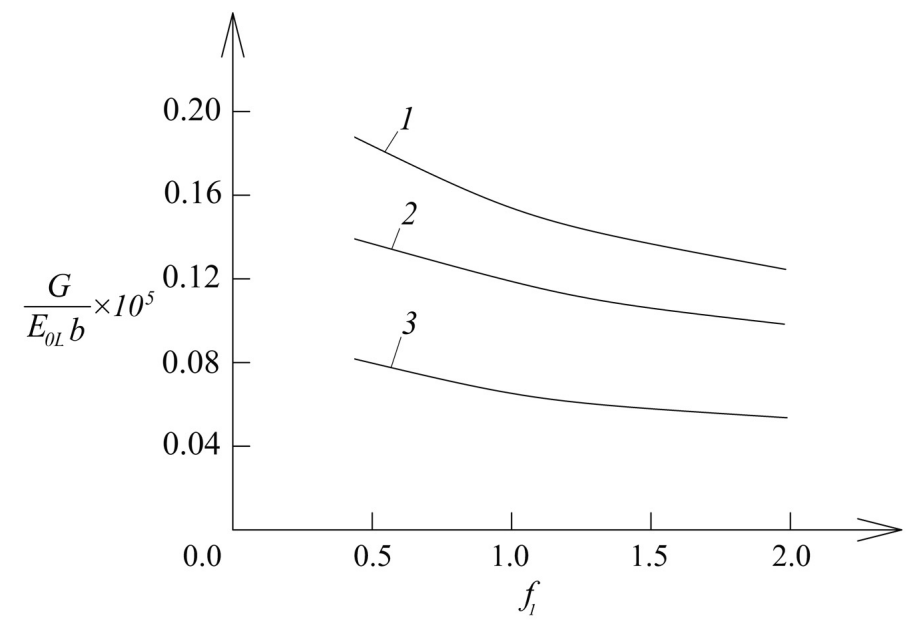

Fig. 4. The strain energy release rate in non-dimensional form presented as a function of $f_{1}$ (curve 1 - at $h_{1} / 2 h=0.25$, curve $2-$ at $h_{1} / 2 h=0.50$ and curve $3-$ at $\left.h_{1} / 2 h=0.75\right)$ 
The results obtained are presented in non-dimensional form by using the formula $G_{N}=$ $G /\left(E_{0 L} b\right)$. The influences of material inhomogeneity along the height and length of the beam, non-linear mechanical behaviour of the material, crack location along the beam height and the crack length on the fracture are analyzed. The crack location along the beam height is characterized by $h_{1} / 2 h$ ratio. The crack length is characterized by $a / l_{1}$ ratio. It is assumed that $b=0.007 \mathrm{~m}, h=0.001 \mathrm{~m}, l_{1}=0.045 \mathrm{~m}, l_{2}=0.005 \mathrm{~m}$ and $F=15 \mathrm{~N}$.

The strain energy release rate in non-dimensional form is presented as a function of $f_{1}$ in Fig. 4 at three $h_{1} / 2 h$ ratios. It is assumed that $H_{0 L} / E_{0 L}=0.6, n_{0 L}=0.5, f_{2} / f_{1}=0.6$, $f_{3} / f_{1}=0.2, r_{1} / f_{1}=0.7, r_{2} / f_{1}=0.6, r_{3} / f_{1}=0.1$ and $a / l_{1}=0.25$. The curves in Fig. 4 . indicate that the strain energy release rate decreases with increasing of $f_{1}$. Fig. 4 shows also that increase of $h_{1} / 2 h$ ratio leads to decrease of the strain energy release rate (this behaviour is due to increase of the stiffness of the lower crack arm).

Fig. 5 shows the strain energy release rate in non-dimensional form as a function of $H_{0 L} / E_{0 L}$ ratio at $h_{1} / 2 h=0.25$ for three values of $r_{1}$. One can observe in Fig. 5 that the strain energy release rate decreases with increasing of $H / E_{0 L}$ ratio. It can also be observed that increase of $r_{1}$ leads to decrease of the strain energy release rate (Fig. 5).

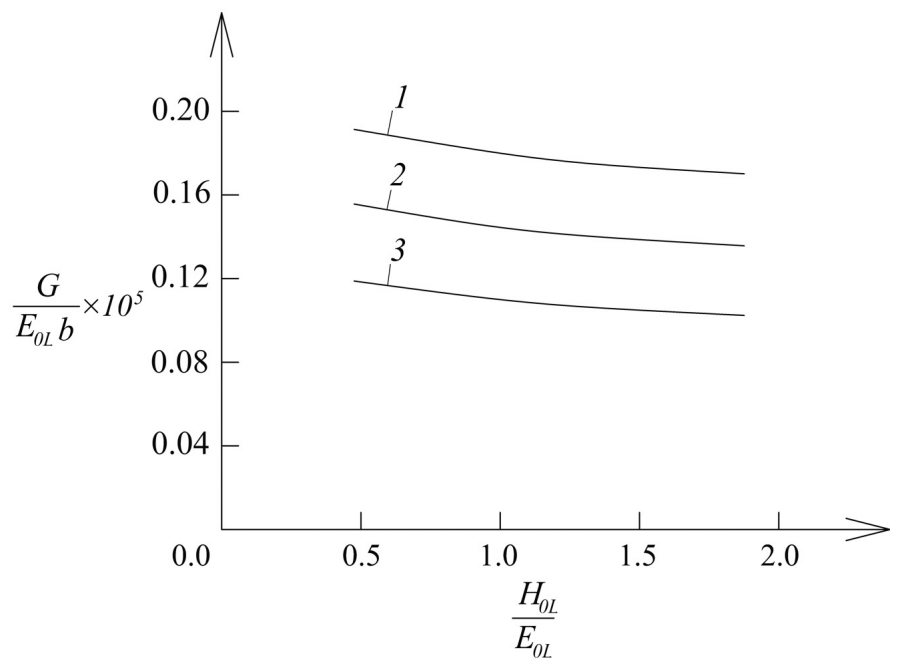

Fig. 5. The strain energy release rate in non-dimensional form presented as a function of $H_{0 L} / E_{0 L}$ ratio (curve $1-$ at $r_{1}=0.5$, curve $2-$ at $r_{1}=1.0$ and curve $3-$ at $r_{1}=2.00$ )

The strain energy release rate in non-dimensional form is presented as a function of $a / l_{1}$ ratio in Fig. 6 at $h_{1} / 2 h=0.25$. It can be observed in Fig. 6 that the strain energy release rate decreases with increasing of $a / l_{1}$ ratio.

This founding is attributed to the fact that the modulus of elasticity in the beam cross-section in which the crack tip is located increases with increasing the crack length. The strain energy release rate derived assuming linear-elastic behaviour of the material is also presented in Fig. 6 . The linear-elastic elastic solution to the strain energy release rate is obtained by substituting of $H_{j} \rightarrow \infty$ in (60) since at $H_{j} \rightarrow \infty$ the Ramberg-Osgood stress-strain relation (7) transforms in the Hooke's law. One can observe in Fig. 6 that the material non-linearity leads to increase of the strain energy release rate.

The effect of $f_{2}$ is also investigated. For this purpose, the strain energy release rate in nondimensional form is presented as a function of $f_{2}$ in Fig. 7 at three values of $f_{3}$ assuming that $h_{1} / 2 h=0.25$ and $a / l_{1}=0.25$. 


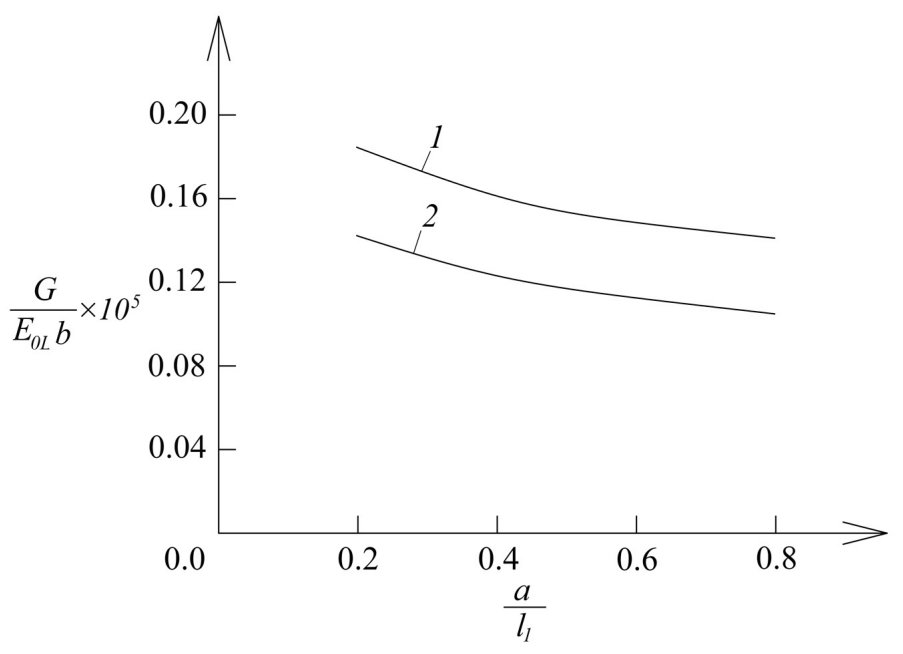

Fig. 6. The strain energy release rate in non-dimensional form presented as a function of $a / l_{1}$ ratio (curve 1 - at non-linear elastic mechanical behaviour of the material and curve 2 - at linear-elastic behaviour)

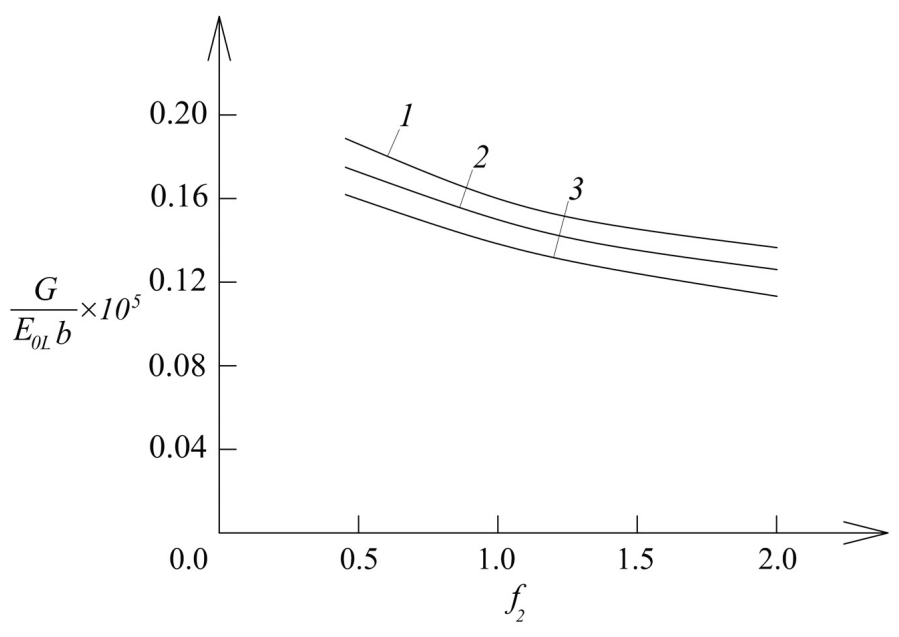

Fig. 7. The strain energy release rate in non-dimensional form presented as a function $f_{2}$ (curve 1 - at $f_{3}=0.1$, curve $2-$ at $f_{3}=0.3$ and curve $3-$ at $f_{3}=0.5$ )

Fig. 7 shows that the strain energy release rate decreases with increasing of $f_{2}$ and $f_{3}$.

The influence of $r_{2}$ and $r_{3}$ on the longitudinal fracture is elucidated in Fig. 8 where the strain energy release rate in non-dimensional form is presented as a function of $r_{2}$ at three values of $r_{3}$. The curves in Fig. 8 indicate that the strain energy release rate decreases with increasing of $r_{2}$ and $r_{3}$.

\section{Conclusion}

An analytical approach for analyzing longitudinal fracture in beams which exhibit material inhomogeneity along the thickness and longitudinal directions is developed. It is assumed that the material has non-linear mechanical behaviour which is modelled by the Ramberg-Osgood stress-strain relation. It is assumed also that the three material properties which are involved in the Ramberg-Osgood relation vary continuously in thickness and longitudinal directions of the 


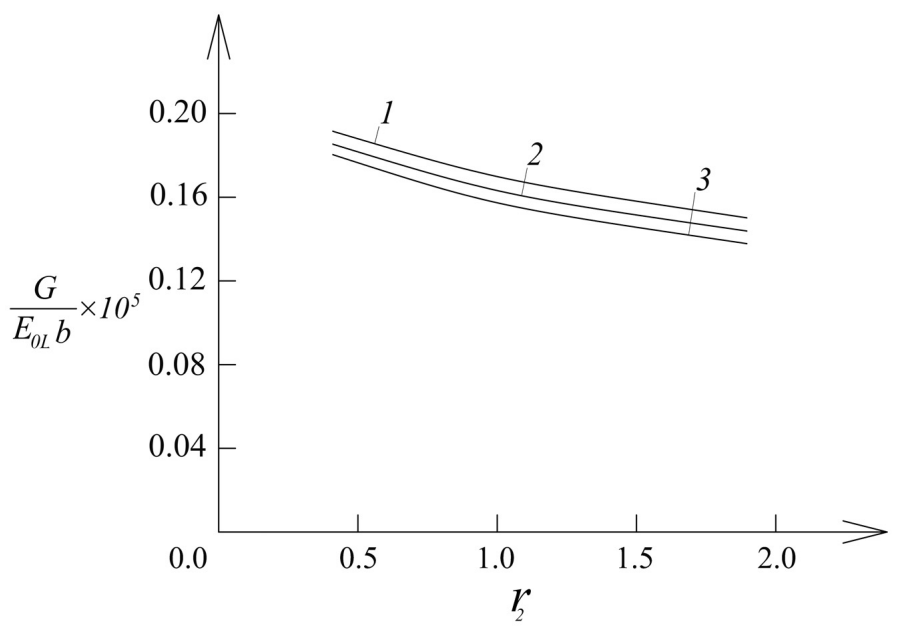

Fig. 8. The strain energy release rate in non-dimensional form presented as a function of $r_{2}$ (curve 1 - at $r_{3}=0.2$, curve $2-$ at $r_{3}=0.3$ and curve $3-$ at $r_{3}=0.4$ )

beam (exponential laws are used for describing the variation of the properties). A solution to the strain energy release rate is derived by analyzing the balance of the energy for general loading scheme. The solution is applicable for a longitudinal crack located arbitrary along the beam height. The strain energy release rate is obtained also by differentiating the complementary strain energy with respect to the crack area for verification. Parametric investigations of the longitudinal fracture are performed. Effects of material inhomogeneity in height and length directions of the beam, non-linear mechanical behaviour of the material, crack location along the beam height and crack length on the longitudinal fracture are elucidated. It is found that the strain energy release rate decreases with increasing of $f_{1}, f_{2}, f_{3}, r_{1}, r_{2}$ and $r_{3}$ (these material properties control the material gradient in the thickness and longitudinal directions). The analysis reveals that the strain energy release rate decreases with increasing of the height of the lower crack arm. It is found also that the strain energy release rate decreases with increasing of the crack length (this founding is attributed to the fact that the modulus of elasticity in the crosssection in which the crack tip is located increases with increasing of the crack length). The strain energy release rate calculated by the solution derived in the present paper has to be compared with the critical value of the strain energy release rate, $G_{c}$, known as fracture toughness in order to check for crack growth. However, it should be mentioned that $G_{c}$ is a function of $x$ and $z$ coordinates since the material under consideration is continuously inhomogeneous along the thickness and longitudinal directions of the beam. Therefore, $G_{c}$ has to be measured by performing experimental testing of beam specimens with longitudinal crack of different lengths located at various positions along the thickness of the beam.

\section{References}

[1] Bohidar, S. K., Sharma, R., Mishra, P. R., Functionally graded materials: A critical review, International Journal of Research 1 (2014) 289-301.

[2] Carpinteri, A., Pugno, N., Cracks in re-entrant corners in functionally graded materials, Engineering Fracture Mechanics 73 (2006) 1279-1291. https://doi.org/10.1016/j.engfracmech.2006.01.008

[3] Erdogan, F., Fracture mechanics of functionally graded materials, Composites Engineering 5 (1995) 753-770. https://doi.org/10.1016/0961-9526(95)00029-M 
[4] Gasik, M. M., Functionally graded materials: Bulk processing techniques, International Journal of Materials and Product Technology 39 (2010) 20-29. https://doi.org/10.1504/IJMPT.2010.034257

[5] Dowling, N., Mechanical behavior of materials, Pearson, 2007.

[6] Joshi, P. V., Jain, N. K., Ramtekkar, G. D., Analytical modelling for vibration analysis of thin rectangular orthotropic/functionally graded plates with an internal crack, Journal of Sound and Vibration 344 (2015) 377-398. https://doi.org/10.1016/j.jsv.2015.01.026

[7] Koizumi, M., The concept of FGM, Ceramic Transactions, Functionally Gradient Materials 34 (1993) 3-10.

[8] Neubrand, A., Rödel, J., Gradient materials: An overview of a novel concept, Zeitschrift Fur Metallkunde 88(5) (1997) 358-371.

[9] Ozturk, M., Erdogan, F., Mode I crack problem in an inhomogeneous orthotropic medium, International Journal of Engineering Science 35(9) (1997) 869-883.

https://doi.org/10.1016/S0020-7225(97)80005-5

[10] Parton, V.Z., Fracture mechanics, Science, 1990.

[11] Rizov, V.I., An analytical solution to the strain energy release rate of a crack in functionally graded nonlinear elastic beams, European Journal of Mechanics A/Solids 65 (2017) 301-312. https://doi.org/10.1016/j.euromechsol.2017.04.005

[12] Rizov, V. I., Analytical study of elastic-plastic longitudinal fracture in a functionally graded beam, Strength, Fracture and Complexity 10 (2017) 11-22. https://doi.org/10.3233/SFC-170197

[13] Rizov, V.I., Delamination in multi-layered functionally graded beams - an analytical study by using the Ramberg-Osgood equation, Structural Integrity and Life 18 (2018) 70-76.

[14] Rizov, V. I., Lengthwise fracture analyses of functionally graded beams by the Ramberg-Osgood equation, Engineering Review 38 (2018) 309-320. https://doi.org/10.30765/er.38.3.8

[15] Rudih, O.L., Sokolov, G.P., Pahomov, V.L., Introduction to non-linear structural mechanics, IASV, Moscow, 1998.

[16] Tilbrook, M. T., Moon, R. J., Hoffman, M., Crack propagation in graded composites, Composite Science and Technology 65 (2005) 201-220. https://doi.org/10.1016/j.compscitech.2004.07.004

[17] Yang, J., Chen, Y., Xiang, Y., Jia, X.L., Free and forced vibration of cracked inhomogeneous beams under an axial force and a moving load, Journal of Sound and Vibration 312 (2008) 166-181. https://doi.org/10.1016/j.jsv.2007.10.034

\section{Appendix}

In order to verify (60), the strain energy release rate is derived also by using the following formula [12]:

$$
G=\frac{\mathrm{d} U^{*}}{b d a}
$$

where $\mathrm{d} U^{*}$ is the change of the complementary strain energy, $d a$ is an infinitesimal increase of the crack length.

The complementary strain energy is written as

$$
U^{*}=U_{1}^{*}+U_{2}^{*}+U_{3}^{*},
$$

where $U_{1}^{*}, U_{2}^{*}$ and $U_{3}^{*}$ are the complementary strain energies cumulated in the lower crack arm and portions $I I-I I I$ and $I I I-I V$ of the beam, respectively.

The complementary strain energy in the lower crack arm is derived as

$$
U_{1}^{*}=b \int_{0}^{a} \int_{-\frac{h_{1}}{2}}^{\frac{h_{1}}{2}} u_{01}^{*} \mathrm{~d} x \mathrm{~d} z_{1},
$$


where the complementary strain energy density in the lower crack arm $u_{01}^{*}$ is calculated by the formula [14]

$$
u_{01}^{*}=\frac{\sigma^{2}}{2 E}+\frac{n \sigma^{\frac{1+n}{n}}}{(1+n) H^{\frac{1}{n}}} .
$$

The complementary strain energies in portions $I I-I I I$ and $I I I-I V$ of the beam are expressed as

$$
\begin{aligned}
& U_{2}^{*}=b \int_{a}^{l_{1}} \int_{-h}^{h} u_{02}^{*} \mathrm{~d} x \mathrm{~d} z_{2}, \\
& U_{3}^{*}=b \int_{l_{1}}^{l_{1}+l_{2}} \int_{-h}^{h} u_{03}^{*} \mathrm{~d} x \mathrm{~d} z_{3},
\end{aligned}
$$

respectively.

By substituting of (62), (63), (65) and (66) in (61), one derives

$$
G=\int_{-\frac{h_{1}}{2}}^{\frac{h_{1}}{2}} u_{01}^{*} \mathrm{~d} z_{1}-\int_{-h}^{h} u_{02}^{*} \mathrm{~d} z_{2},
$$

where $u_{01}^{*}$ and $u_{02}^{*}$ are found at $x=a$. The strain energy release rate calculated by (67) is exact match of that found by (60) which verifies the present analysis. Also, an analytical proof that (67) is identical with (60) is presented. For this purpose, $u_{01}^{*}$ is written as

$$
u_{01}^{*}=\sigma \varepsilon-u_{01}
$$

Formula (68) follows from the fact that the complementary strain energy density is equal to the area that supplements the area enclosed by the stress-strain curve to a rectangle. By using (68), the first term of the right-hand side of (67) is expressed as

$$
\int_{-\frac{h_{1}}{2}}^{\frac{h_{1}}{2}} u_{01}^{*} \mathrm{~d} z_{1}=\int_{-\frac{h_{1}}{2}}^{\frac{h_{1}}{2}} \sigma \varepsilon \mathrm{d} z_{1}-\int_{-\frac{h_{1}}{2}}^{\frac{h_{1}}{2}} u_{01} \mathrm{~d} z_{1} .
$$

Since the strain is distributed linearly along the thickness, the first integral in the right-hand side of (69) can be trasformed as

$$
\int_{-\frac{h_{1}}{2}}^{\frac{h_{1}}{2}} \sigma \kappa_{1} z_{1} \mathrm{~d} z_{1}=\frac{\kappa_{1}}{b} b \int_{-\frac{h_{1}}{2}}^{\frac{h_{1}}{2}} \sigma z_{1} \mathrm{~d} z_{1} .
$$

The stress $\sigma$ is connected to the bending moment $\mathrm{Fl}_{2}$ in the lower crack arm by the following equation for equilibrium:

$$
F l_{2}=b \int_{-\frac{h_{1}}{2}}^{\frac{h_{1}}{2}} \sigma z_{1} \mathrm{~d} z_{1} .
$$

By combining of (70) and (71), formula (69) is rewritten as

$$
\int_{-\frac{h_{1}}{2}}^{\frac{h_{1}}{2}} u_{01}^{*} \mathrm{~d} z_{1}=\frac{\kappa_{1}}{b} F l_{2}-\int_{-\frac{h_{1}}{2}}^{\frac{h_{1}}{2}} u_{01} \mathrm{~d} z_{1} .
$$


Analogically, the second integral in (67) is transformed as

$$
\int_{-h}^{h} u_{02}^{*} \mathrm{~d} z_{2}=\frac{\kappa_{2}}{b} F l_{2}-\int_{-h}^{h} u_{02} \mathrm{~d} z_{1} .
$$

By substituting of (72) and (73) in (67), one obtains expression for the strain energy release rate that is exact match of (60). This fact is also a verification of the solution to the strain energy release rate derived in the present paper. 